\title{
EXISTENCE OF INVERSES AND SQUARE ROOTS IN LOCALLY BANACH SEMIGROUPS WITH IDENTITY ${ }^{1}$
}

\author{
ROBERT C. ESLINGER
}

\begin{abstract}
Let $S$ be a multiplicative topological semigroup with identity $e$. Suppose $D$ is an open subset containing $e$ and $h$ is a homeomorphism from $D$ onto a Banach space $B$ with $h(e)=0$. Define the function $P$ by $P(x, y)=h\left[h^{-1}(x) \cdot h^{-1}(y)\right]$. A new implicit function theorem is applied to the function $P$ to show the existence of inverses and square roots of elements in a neighborhood of the identity. It is assumed that $P$ satisfies the following condition: There exist a one-one function $A$ from a subset of $B$
\end{abstract} into $B$ and positive numbers $r, M$, and $c$ such that

(i) if $\|x\|<r$ then $x \in \operatorname{dom}\left(A^{-1}\right)$ and $\left\|A^{-1}(x)\right\| \leqslant M\|x\|$,

(ii) $c M<1$, and

(iii) if $\left\|x_{i}\right\|,\left\|y_{i}\right\|<r(i=1,2)$ then $\left(x_{i}, y_{j}\right) \in \operatorname{dom}(P)(i, j=1,2)$,

$$
\left\|P\left(x_{1}, y_{1}\right)-P\left(x_{1}, y_{2}\right)-A\left(y_{1}-y_{2}\right)\right\| \leqslant c\left\|y_{1}-y_{2}\right\| \text {, }
$$

and

$$
\left\|P\left(x_{1}, y_{1}\right)-P\left(x_{2}, y_{1}\right)-A\left(x_{1}-x_{2}\right)\right\| \leqslant c\left\|x_{1}-x_{2}\right\| .
$$

1. Introduction. Suppose $S$ is a multiplicative topological semigroup with an identity contained in an open subset homeomorphic to a Banach space. The purpose of this paper is to state sufficient conditions under which there exist inverses and square roots for elements near the identity (see the hypothesis of Theorem 2). In the finite dimensional case it is known [4] that no additional conditions are necessary. However, Holmes has shown [2] that this is not true in the general Banach space setting. Birkhoff [1] has established sufficient conditions in this case and that result is improved here.

In the spirit of Hilbert's fifth problem ${ }^{2}$ the assumptions made on the semigroup do not require differentiability of the semigroup operation. If one assumes multiplication is continuously differentiable, an application of the classical implicit function theorem yields inverses for elements in a neighborhood of the identity. The author presents a new implicit function theorem the proof of which uses a technique of successive approximation analogous to Newton's method. This theorem along with its counterpart, an inverse function theorem, is used then to give the desired results in the semigroup.

Presented to the Society, September 29, 1971 under the title An implicit function theorem with applications to locally Banach semigroups with identity; received by the editors February 1, 1975.

AMS (MOS) subject classifications (1970). Primary 22A15, 47H10, 58C15.

Key words and phrases. Topological semigroup, Banach manifold, implicit function theorem.

${ }^{1}$ This paper is based on the author's thesis prepared under the supervision of Professor $\mathbf{J}$. W. Neuberger at Emory University.

${ }^{2}$ Montgomery and Zippin [3] present an extensive treatment of Hilbert's fifth problem and its solution in the locally compact case. 
It should be noted also that a degree of nonlinearity is introduced in the assumptions on the semigroup operation. This is seen most graphically when one compares the hypothesis of Theorem 2 with Birkhoff's definition of an analytical group nucleus.

2. Implicit function theorem. Let $B$ be a Banach space. If $x \in B$ and $\delta>0$ denote by $N(x ; \delta)$ the symmetric open neighborhood about $x$ with radius $\delta$.

THEOREM 1. Let each of $D$ and $G$ be an open subset of $B,\left(x_{0}, y_{0}\right) \in D \times G$, and $P: D \times G \rightarrow B$ be a continuous function with $P\left(x_{0}, y_{0}\right)=0$. Suppose there exist a one-one function $A$ from $a$ subset of $B$ into $B$ and positive numbers $r, M$, and $c$ so that

(i) if $\|x\|<r$ then $x \in \operatorname{dom}\left(A^{-1}\right)$ and $\left\|A^{-1}(x)\right\| \leqslant M\|x\|$,

(ii) $c M<1$, and

(iii) if $x \in N\left(x_{0} ; r\right)$ and each of $y_{1}, y_{2} \in N\left(y_{0} ; r\right)$ then $\left(x, y_{i}\right) \in D \times G$ $(i=1,2)$ and

$$
\left\|P\left(x, y_{1}\right)-P\left(x, y_{2}\right)-A\left(y_{1}-y_{2}\right)\right\| \leqslant c\left\|y_{1}-y_{2}\right\| .
$$

Then there is a connected open subset $D^{\prime}$ of $D$ containing $x_{0}$ and a unique continuous function $u: D^{\prime} \rightarrow G$ such that $u\left(x_{0}\right)=y_{0}$ and $P(x, u(x))=0$ for all $x \in D^{\prime}$.

Before proceeding to the proof a Lemma is stated under the hypothesis of the theorem.

LEMMA. There is a positive number $\delta \leqslant r$ and a connected open subset $D^{\prime}$ of $D$ containing $x_{0}$ such that (1) $\left\|A\left(y_{1}-y_{2}\right)\right\|<r$ whenever each of $y_{1}, y_{2} \in$ $N\left(y_{0} ; \delta\right)$, and (2) if $x \in D^{\prime}$ then $x \in N\left(x_{0} ; r\right)$ and the sequence $\left\{y_{k}\right\}$ defined inductively by

$$
y_{k}=y_{k-1}-A^{-1}\left[P\left(x, y_{k-1}\right)\right]
$$

converges to a point $y \in N\left(y_{0} ; \delta\right)$.

Proof. Suppose the hypothesis of Theorem 1. Let

$$
0<\delta \leqslant \min \{(1-c M) r / 2 c, r\}
$$

such that if $x \in N\left(x_{0} ; \delta\right)$ and $y \in N\left(y_{0} ; \delta\right)$ then

$$
\|P(x, y)\|=\left\|P(x, y)-P\left(x_{0}, y_{0}\right)\right\|<r c M / 2 \text {. }
$$

Suppose each of $y_{1}, y_{2} \in N\left(y_{0} ; \delta\right)$. Using condition (iii),

$$
\left\|A\left(y_{1}-y_{2}\right)\right\| \leqslant\left\|P\left(x_{0}, y_{1}\right)-P\left(x_{0}, y_{2}\right)\right\|+c\left\|y_{1}-y_{2}\right\|<2 \frac{r c M}{2}+2 c \delta \leqslant r
$$

and ( 1 ) is established.

Let $0<\delta^{\prime} \leqslant \delta$ such that if $x \in N\left(x_{0} ; \delta^{\prime}\right)$ then $\left\|P\left(x, y_{0}\right)\right\|<c(1-c M) \delta$. Define $D^{\prime}=N\left(x_{0} ; \delta^{\prime}\right)$, which is a subset of $N\left(x_{0} ; r\right)$, and suppose $x \in D^{\prime}$. Since $\left\|P\left(x, y_{0}\right)\right\|<r, y_{1}$ is well defined by $(*)$, and

$$
\left\|y_{1}-y_{0}\right\| \leqslant M\left\|P\left(x, y_{0}\right)\right\|<c M(1-c M) \delta .
$$

Now suppose $n$ is a positive integer and each of $y_{1}, y_{2}, \ldots, y_{n}$ has been defined by $(*)$ such that 


$$
\left\|y_{k}-y_{k-1}\right\|<(c M)^{k}(1-c M) \delta, \quad k=1,2, \ldots, n .
$$

Then for each $k=1,2, \ldots, n$,

$$
\left\|y_{k}-y_{0}\right\| \leqslant \sum_{i=1}^{k}\left\|y_{i}-y_{i-1}\right\|<(1-c M) \delta \sum_{i=1}^{k}(c M)^{i}<(c M) \delta
$$

from which it follows that $\left\|P\left(x, y_{k}\right)\right\|<r c M / 2<r$. So $y_{n+1}$ is well defined by (*). Furthermore, noting from (*) that $P\left(x, y_{n-1}\right)=A\left(y_{n-1}-y_{n}\right)$,

$$
\begin{aligned}
\left\|y_{n+1}-y_{n}\right\| & \leqslant M\left\|P\left(x, y_{n}\right)\right\|=M\left\|P\left(x, y_{n-1}\right)-P\left(x, y_{n}\right)-A\left(y_{n-1}-y_{n}\right)\right\| \\
& \leqslant c M\left\|y_{n-1}-y_{n}\right\|<(c M)^{n+1}(1-c M) \delta .
\end{aligned}
$$

It is evident that $\left\{y_{k}\right\}$ is a Cauchy sequence and therefore converges to a point $y$. Moreover $\left\|y-y_{0}\right\| \leqslant(c M) \delta<\delta$, and the Lemma is proved.

Proof of Theorem 1. Choose $\delta$ and $D^{\prime}$ as in the Lemma and define the function $u: D^{\prime} \rightarrow G$ such that for each $x \in D^{\prime}, u(x)$ is the limit of the sequence defined by $(*)$ with respect to $x$.

Since $P\left(x_{0}, y_{0}\right)=0$ and (i) implies that $A(0)=0$, it follows that $u\left(x_{0}\right)=y_{0}$. Now suppose $x \in D^{\prime}$ and $\left\{y_{k}\right\}$ is the sequence defined by $(*)$ which converges to $u(x)$. Condition (iii) implies $A$ is continuous at the origin, so $P\left(x, y_{k}\right)=A\left(y_{k}-y_{k+1}\right) \rightarrow 0$ as $k \rightarrow \infty$. Therefore continuity of $P$ gives that $P(x, u(x))=0$.

To show $u$ is continuous let each of $x_{1}, x_{2} \in D^{\prime}$. From the Lemma $u\left(x_{i}\right) \in N\left(y_{0} ; \delta\right)$ for $i=1,2$; therefore

$$
\begin{aligned}
\left\|u\left(x_{1}\right)-u\left(x_{2}\right)\right\|= & \left\|A^{-1}\left[A\left(u\left(x_{1}\right)-u\left(x_{2}\right)\right)\right]\right\| \leqslant M\left\|A\left(u\left(x_{1}\right)-u\left(x_{2}\right)\right)\right\| \\
\leqslant & M\left\|P\left(x_{2}, u\left(x_{1}\right)\right)-P\left(x_{2}, u\left(x_{2}\right)\right)-A\left(u\left(x_{1}\right)-u\left(x_{2}\right)\right)\right\| \\
& +M\left\|P\left(x_{1}, u\left(x_{1}\right)\right)-P\left(x_{2}, u\left(x_{1}\right)\right)\right\| \\
\leqslant & c M\left\|u\left(x_{1}\right)-u\left(x_{2}\right)\right\|+M\left\|P\left(x_{1}, u\left(x_{1}\right)\right)-P\left(x_{2}, u\left(x_{1}\right)\right)\right\| .
\end{aligned}
$$

Hence

$$
\left\|u\left(x_{1}\right)-u\left(x_{2}\right)\right\| \leqslant \frac{M}{1-c M}\left\|P\left(x_{1}, u\left(x_{1}\right)\right)-P\left(x_{2}, u\left(x_{1}\right)\right)\right\| .
$$

By continuity of $P$, then, $u$ is continuous.

It remains to be shown that $u$ is unique. Suppose $v: D^{\prime} \rightarrow G$ is a continuous function satisfying $v\left(x_{0}\right)=y_{0}$ and $P(x, v(x))=0$ for all $x \in D^{\prime}$. Let $E$ $=\left\{x \in D^{\prime}: u(x)=v(x)\right\}$. Clearly $x_{0} \in E$ and, since each of $u$ and $v$ is continuous, $E$ is closed relative to $D^{\prime}$. $E$ is also open for suppose $x \in E$. Recalling that $\left\|v(x)-y_{0}\right\|=\left\|u(x)-y_{0}\right\|<\delta$ choose $\delta^{\prime}>0$ such that $z \in$ $N\left(x ; \delta^{\prime}\right)$ implies $z \in D^{\prime}$ and $\left\|v(z)-y_{0}\right\|<\delta$. Now if $z \in N\left(x ; \delta^{\prime}\right)$ then

$$
\begin{aligned}
\|u(z)-v(z)\| & \leqslant M\|A(u(z)-v(z))\| \\
& =M\|P(z, u(z))-P(z, v(z))-A(u(z)-v(z))\| \\
& \leqslant c M\|u(z)-v(z)\|
\end{aligned}
$$

which implies that $u(z)=v(z)$. Since $D^{\prime}$ is connected, $E=D^{\prime}$ and the theorem is proved.

Corollary. Suppose $G$ is an open subset of $B, y_{0} \in G$, and $f: G \rightarrow B$ is $a$ 
continuous function for which there exist a one-one function $A$ from a subset of $B$ into $B$ and positive numbers $r, M$, and $c$ such that

(i) if $\|x\|<r$ then $x \in \operatorname{dom}\left(A^{-1}\right)$ and $\left\|A^{-1}(x)\right\| \leqslant M\|x\|$,

(ii) $c M<1$, and

(iii) if each of $y_{1}, y_{2} \in N\left(y_{0} ; r\right)$ then $y_{i} \in G(i=1,2)$ and

$$
\left\|f\left(y_{1}\right)-f\left(y_{2}\right)-A\left(y_{1}-y_{2}\right)\right\| \leqslant c\left\|y_{1}-y_{2}\right\| \text {. }
$$

Then there exists an open subset $G^{\prime}$ of $G$ containing $y_{0}$ such that the restriction of $f$ to $G^{\prime}$ is a homeomorphism from $G^{\prime}$ onto an open set containing $f\left(y_{0}\right)$.

Proof. Let $x_{0}=f\left(y_{0}\right)$. Define the function $P: B \times G \rightarrow B$ by $P(x, y)$ $=f(y)-x$. Clearly $P$ satisfies the hypothesis of Theorem 1 . Choose $0<\delta$ $\leqslant r$ to satisfy (1) of the Lemma and suppose each of $y_{1}, y_{2} \in N\left(y_{0} ; \delta\right)$. Then

$$
\begin{aligned}
\left\|y_{1}-y_{2}\right\| & \leqslant M\left\|A\left(y_{1}-y_{2}\right)\right\| \\
& \leqslant M\left\|f\left(y_{1}\right)-f\left(y_{2}\right)-A\left(y_{1}-y_{2}\right)\right\|+M\left\|f\left(y_{1}\right)-f\left(y_{2}\right)\right\| \\
& \leqslant c M\left\|y_{1}-y_{2}\right\|+M\left\|f\left(y_{1}\right)-f\left(y_{2}\right)\right\| .
\end{aligned}
$$

Therefore

$$
\left\|y_{1}-y_{2}\right\| \leqslant \frac{M}{1-c M}\left\|f\left(y_{1}\right)-f\left(y_{2}\right)\right\| .
$$

It follows that $f$ is one-one on $N\left(y_{0} ; \delta\right)$.

Let $D$ be an open set containing $x_{0}$ and $u: D \rightarrow G$ a continuous function such that $u\left(x_{0}\right)=y_{0}$ and $P(x, u(x))=0$ for all $x \in D$. Let $G^{\prime}=u[D] \cap$ $N\left(y_{0} ; \delta\right)$. Now $f$ is one-one on $G^{\prime}$ and, according to the definition of $P$, $f(u(x))=x$ for all $x \in D$. Hence the inverse of the restriction of $f$ to $G^{\prime}$ is $u$ restricted to $f\left[G^{\prime}\right]$.

To show $G^{\prime}$ is open suppose $y \in G^{\prime}$ and let $u(x)=y$. Choose $\varepsilon>0$ such that if $\left\|x_{1}-x\right\|<\varepsilon$ then $x_{1} \in D$ and $u\left(x_{1}\right) \in N\left(y_{0} ; \delta\right)$. Choose $\delta^{\prime}>0$ such that if $y_{1} \in N\left(y ; \delta^{\prime}\right)$ then $y_{1} \in N\left(y_{0} ; \delta\right)$ and $\left\|f\left(y_{1}\right)-f(y)\right\|=\| f\left(y_{1}\right)-$ $x \|<\varepsilon$. Now if $y_{1} \in N\left(y ; \delta^{\prime}\right)$ then each of $u\left(f\left(y_{1}\right)\right)$ and $y_{1}$ is in $N\left(y_{0} ; \delta\right)$ with $f\left[u\left(f\left(y_{1}\right)\right)\right]=f\left(y_{1}\right)$ which implies that $y_{1}=u\left(f\left(y_{1}\right)\right)$. Hence $y_{1} \in G^{\prime}$.

Since each of $D$ and $G^{\prime}$ is open and $u$ is continuous, $f\left[G^{\prime}\right]$ is an open set containing $f\left(y_{0}\right)$ and the Corollary is proved.

3. Applications to semigroups. Let $S$ be a multiplicative topological semigroup with identity $e$ which is locally Banach at $e$. Let $D$ be an open subset of $S$ containing $e$ and $h$ a homeomorphism from $D$ onto a Banach space $B$. Assume $h(e)=0$. Define the function $P$ such that $\operatorname{dom}(P)$ is the set of all ordered pairs $(x, y) \in B \times B$ for which $h^{-1}(x) \cdot h^{-1}(y) \in D$, and for each $(x, y) \in \operatorname{dom}(P)$,

$$
P(x, y)=h\left[h^{-1}(x) \cdot h^{-1}(y)\right] .
$$

It is a consequence of the definition that $\operatorname{dom}(P)$ is open and $P$ is continuous.

TheOREM 2. Suppose there exist a one-one function $A$ from a subset of $B$ into $B$ and positive numbers $r, M$, and $c$ such that

(i) if $\|x\|<r$ then $x \in \operatorname{dom}\left(A^{-1}\right)$ and $\left\|A^{-1}(x)\right\| \leqslant M\|x\|$,

(ii) $c M<1$, and 
(iii) if each of $x_{1}, x_{2}, y_{1}, y_{2} \in N(0 ; r)$ then $\left(x_{i}, y_{j}\right) \in \operatorname{dom}(P)(i, j=1,2)$,

$$
\left\|P\left(x_{1}, y_{1}\right)-P\left(x_{1}, y_{2}\right)-A\left(y_{1}-y_{2}\right)\right\| \leqslant c\left\|y_{1}-y_{2}\right\|,
$$

and

$$
\left\|P\left(x_{1}, y_{1}\right)-P\left(x_{2}, y_{1}\right)-A\left(x_{1}-x_{2}\right)\right\| \leqslant c\left\|x_{1}-x_{2}\right\| .
$$

Then there is an open subset $D^{\prime}$ of $D$ containing e, each member of which has an inverse and the set of all invertible elements in $S$ is a topological group open in $S$.

Proof. Let $Q$ be the function defined on $N(0 ; r) \times N(0 ; r)$ by $Q(x, y)=P(y, x)$. Then each of $P$ and $Q$ satisfies the hypothesis of Theorem 1 at the point $(0,0)$. Therefore let each of $U$ and $V$ be an open subset of $N(0 ; r)$ containing 0 and let each of $u$ and $v$ be a continuous function such that (1) $u: U \rightarrow N(0 ; r)$ and $v: V \rightarrow N(0 ; r),(2) u(0)=v(0)=0$, and (3) $P(x, u(x))=0$ for all $x \in U$ and $Q(x, v(x))=0$ for all $x \in V$. Let $D^{\prime}=h^{-1}[U \cap V]$.

Suppose $\alpha \in D^{\prime}$. Then $h(\alpha) \in U \cap V$, hence

$$
P(h(\alpha), u[h(\alpha)])=Q(h(\alpha), v[h(\alpha)])=0 .
$$

From the definitions of $P$ and $Q$ and the fact that $h$ is one-one it follows that

$$
\alpha \cdot h^{-1}(u[h(\alpha)])=h^{-1}(v[h(\alpha)]) \cdot \alpha=e .
$$

Furthermore,

$$
\begin{aligned}
h^{-1}(u[h(\alpha)]) & =\left[h^{-1}(v[h(\alpha)]) \cdot \alpha\right] \cdot h^{-1}(u[h(\alpha)]) \\
& =h^{-1}(v[h(\alpha)]) \cdot\left[\alpha \cdot h^{-1}(u[h(\alpha)])\right]=h^{-1}(v[h(\alpha)]) .
\end{aligned}
$$

Therefore $\alpha$ has an inverse and the inverse operation on $D^{\prime}$ is $h^{-1}(u(h))$.

Denote by $S(e)$ the set of all invertible elements in $S$ with the relative topology. Clearly $S(e)$ is a group with continuous multiplication. Furthermore, if $\alpha \in S(e)$ then the set $\alpha D^{\prime}=\left\{\alpha \cdot \delta: \delta \in D^{\prime}\right\}$ is an open subset of $S(e)$ containing $\alpha$ and the inverse operation on $\alpha D^{\prime}$ is given by $\beta^{-1}$ $=\left(\alpha^{-1} \beta\right)^{-1} \cdot \alpha^{-1}$ which is continuous since the inverse operation on $D^{\prime}$ is continuous. Therefore $S(e)$ is a topological group open in $S$ and the theorem is proved.

THEOREM 3. Under the hypothesis of Theorem 2 there is an open subset $D^{\prime}$ of $D$ containing e, each member of which has a square root.

Proof. Define the function $f: N(0 ; r) \rightarrow B$ by $f(x)=P(x, x)$. Now $f$ satisfies the hypothesis of the Corollary to Theorem 1 at the origin with respect to the one-one function $2 A$ and the positive numbers $r, M / 2$, and $2 c$. Conditions (i) and (ii) are verified easily. To show (iii) is satisfied suppose each of $y_{1}, y_{2} \in N(0 ; r)$. Then

$$
\begin{aligned}
\left\|f\left(y_{1}\right)-f\left(y_{2}\right)-2 A\left(y_{1}-y_{2}\right)\right\| \leqslant & \left\|P\left(y_{1}, y_{1}\right)-P\left(y_{1}, y_{2}\right)-A\left(y_{1}-y_{2}\right)\right\| \\
& +\left\|P\left(y_{1}, y_{2}\right)-P\left(y_{2}, y_{2}\right)-A\left(y_{1}-y_{2}\right)\right\| \\
& \leqslant 2 c\left\|y_{1}-y_{2}\right\| .
\end{aligned}
$$

Let $G$ be an open subset of $N(0 ; r)$ containing the origin such that $f$ is 
one-one on $G$ and $f[G]$ is open. Let $D^{\prime}=h^{-1}[f[G]]$.

Suppose $\alpha \in D^{\prime}$. Let $x \in G$ such that $f(x)=h(\alpha)$; that is, such that $h\left[h^{-1}(x) \cdot h^{-1}(x)\right]=h(\alpha)$. Then $\alpha=h^{-1}(x) \cdot h^{-1}(x)$ and the theorem is proved.

\section{REFERENCES}

1. Garrett Birkhoff, Analytical groups, Trans. Amer. Math. Soc. 43(1938), 61-101.

2. J. P. Holmes, Partial groupoids on a Banach space, Thesis, Emory Univ., Atlanta, Ga. 1971.

3. D. Montgomery and L. Zippin, Topological transformation groups, Interscience, New York, 1955. MR17, 383.

4. P. S. Mostert and A. L. Shields, Semigroups with identity on a manifold, Trans. Amer. Math. Soc. 91(1959), 380-389. MR21 \#4204.

Department of Mathematics, King College, Bristol, Tennessee 37620

Current address: Department of Mathematics, University of the South, Sewanee, Tennessee 37375 